\title{
GluNet: A Deep Learning Framework For Accurate Glucose Forecasting
}

\author{
Kezhi Li, Chengyuan Liu, Taiyu Zhu, Pau Herrero, Pantelis Georgiou \\ Department of Electronic and Electrical Engineering, Imperial College London, London SW7 2AZ, UK
}

\begin{abstract}
For people with Type 1 diabetes (T1D), forecasting of blood glucose (BG) can be used to effectively avoid hyperglycemia, hypoglycemia and associated complications. The latest continuous glucose monitoring (CGM) technology allows people to observe glucose in real-time. However, an accurate glucose forecast remains a challenge. In this work, we introduce GluNet, a framework that leverages on a personalized deep neural network to predict the probabilistic distribution of short-term (30-60 minutes) future CGM measurements for subjects with T1D based on their historical data including glucose measurements, meal information, insulin doses, and other factors. It adopts the latest deep learning techniques consisting of four components: data pre-processing, label transform/recover, multi-layers of dilated convolution neural network (CNN), and post-processing. The method is evaluated in-silico for both adult and adolescent subjects. The results show significant improvements over existing methods in the literature through a comprehensive comparison in terms of root mean square error (RMSE) $(8.88 \pm 0.77 \mathbf{~ m g} / \mathbf{d L})$ with short time lag $(0.83 \pm 0.40$ minutes) for prediction horizons (PH) $=30$ mins (minutes), and RMSE $(19.90 \pm 3.17 \mathrm{mg} / \mathbf{d L})$ with time lag $(16.43 \pm 4.07$ mins $)$ for $\mathbf{P H}=\mathbf{6 0}$ mins for virtual adult subjects. In addition, GluNet is also tested on two clinical data sets. Results show that it achieves an RMSE $(19.28 \pm 2.76 \mathbf{~ m g / d L})$ with time lag $(8.03 \pm 4.07$ mins) for $\mathbf{P H}=\mathbf{3 0} \mathbf{~ m i n s}$ and an $\mathbf{R M S E}$ $(31.83 \pm 3.49 \mathbf{~ m g} / \mathbf{d L})$ with time lag $(17.78 \pm 8.00 \mathrm{mins})$ for $\mathbf{P H}=\mathbf{6 0}$ mins. These are the best reported results for glucose forecasting when compared with other methods including the neural network for predicting glucose (NNPG), the support vector regression (SVR), the latent variable with exogenous input (LVX), and the auto regression with exogenous input (ARX) algorithm.
\end{abstract}

Index Terms-Deep learning, dilated convolutions, glucose forecasting, continuous glucose monitoring (CGM), diabetes.

\section{INTRODUCTION}

Diabetes is a chronic disease that affects about 415 million people around the world [1]. There are two main types of diabetes: Type-1 diabetes (T1D) and Type-2 diabetes (T2D). For people with T1D, exogenous insulin administration is required to manage BG levels in the target range $(70-180 \mathrm{mg} / \mathrm{dl})$ to avoid hypoglycemia $(<70 \mathrm{mg} / \mathrm{dl})$ or hyperglycemia $(>180 \mathrm{mg} / \mathrm{dl})$. The recent developments in CGM technology [2] provide realtime monitoring of the current glucose, so people can observe their glucose concentration trend visually on devices. It has been proven that CGM technology helps T1D subjects to have a better glycemic control [3], [4] by alerting them when hypoor hyperglycemia events occur [5]-[7].

In recent years many algorithms have been proposed for glucose forecasting [8], such as polynomial models (autoregressive (AR), autoregressive exogenous (ARX), autoregressive moving-average (ARMA)) [9], machine learning models

The work was supported by EPSRC EP/P00993X/1
[10] and latent-variable-based statistical models [11]. Oviedo et al. [8] reviewed the topic comprehensively. In addition, machine learning (ML) techniques were extensively exploited, including support vector regression (SVR) [12], random forest (RF) [13] and grammatical evolution [14]. Deep learning (DL) is a promising ML technique which is still relatively new in this field. DL has potential because it can capture the complex dynamics of systems, particularly when it is difficult to derive the mathematical expressions of the system. DL has improved the state-of-the-art performance significantly in computer vision $(\mathrm{CV})$ and bio-engineering applications [15]. There are a few works to date using DL for glucose forecasting. For instance, in [10], [16] traditional dense neural networks were used but without exploiting the advantages of deep layers. In [17], two latent layer neural networks were adopted for hypo/hyperglycemia prediction. However, this work lacks a comprehensive comparison with other algorithms.

In this paper we introduce GluNet, a framework that leverages deep neural networks (DNN) for forecasting accurate short-time CGM measurements $(\mathrm{PH}=30$ minutes and 60 minutes) using life-style data. These data include historical BG data from CGM, meal intake, insulin dosage. Other data from wearable technology or physiological glucose-insulin regulatory models are optional. GluNet consists of several components, including data preprocessing, label transform/recover, multi-layers of dilated convolution neural network (CNN), gated activations, residual and skip connections. The original idea comes from PixelCNN [18] and Wavenet [19], which are DL models used in $\mathrm{CV}$ and acoustic signal processing. Dilated CNNs are good at processing multi-dimensional long signals with a wider receptive field, and gated activation units capture the non-linearity of the probabilistic relations among several inputs. We modify PixelCNN/Wavenet accordingly to make it appropriate for glucose forecasting, and the DNN becomes a predictive model with fast implementation instead of a generative model. GluNet offers a personalized glucose forecast, and the model can evolve when new personal data are collected. We have evaluated GluNet on 10 simulated virtual T1D adult and adolescent subjects, respectively, generated from the UVA/Padova T1D simulator [20], as well as two clinical dataset including 10 T1D subjects from the ABC4D project using Dexcom ${ }^{T M}$ CGM sensors [21], and 6 T1D subjects from Ohio Dataset [22]. The results show that the proposed framework achieves state-of-the-art glucose forecasting in terms of accuracy and time lag on both in silico and the clinical dataset. 


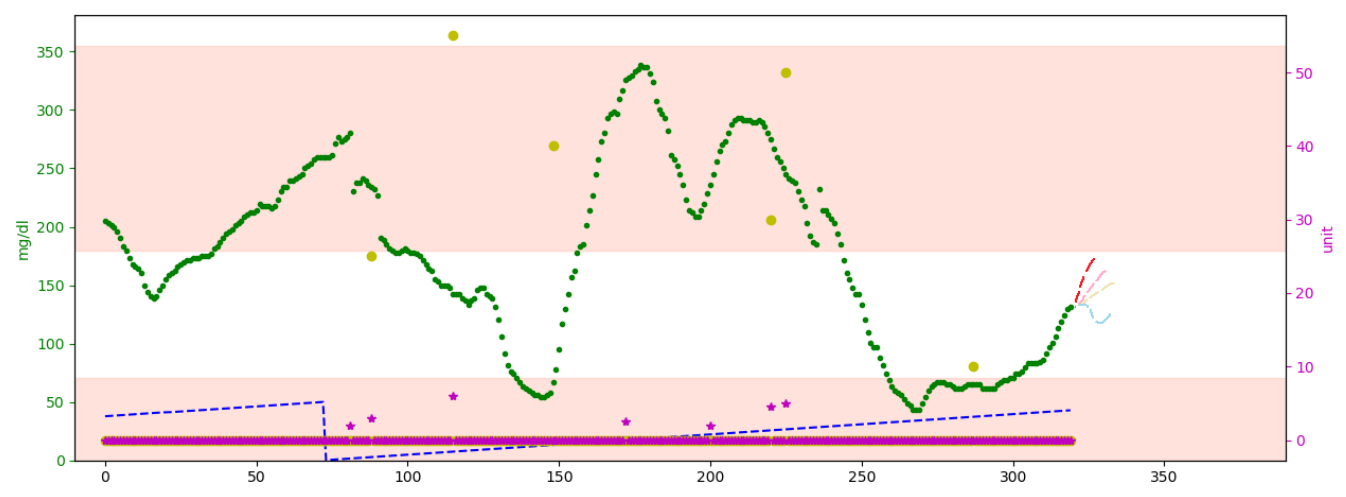

Fig. 1: An illustration of clinical CGM glucose time series and CGM measurements forecasting. $x$-axis stands for glycemic samples; $y$-left-axis represents the blood glucose level (in $\mathrm{mg} / \mathrm{dl}$ ). Green dots are glucose measurements sampled every 5 minutes by CGM in real-time. Purple dots are the insulin dosage (in units) shown on $y$-right-axis. Yellow dots denote the food carbohydrate (in gram) shown on the $y$-right-axis. The blue zigzag line represents the scale of one day length starting from the lowest point (0:00) to the highest point (24:00). The pink area means the hypoglycemia field $(<70 \mathrm{mg} / \mathrm{dl})$ and hyperglycemia field $(>180 \mathrm{mg} / \mathrm{dl})$. Dash lines at the right end of the green dots demonstrate several future forecasting glucose curves.

\section{GLUNET}

\section{A. Problem Formulation}

If a $d$-dimensional $\mathbf{x}_{T}=\left[\mathbf{x}_{1}, \cdots, \mathbf{x}_{T}\right]^{\top} \in \mathbb{R}^{T \times d}$ is the concatenation of historical data and features, where $\mathbf{x}_{t}=\left[\mathbf{m}_{t}, \mathbf{n}_{t}\right]$ consists of $d_{1}$-dimensional historical data and $d_{2}$-dimensional features,

$$
\begin{aligned}
& \mathbf{m}_{t}=\left[m_{t}(1), m_{t}(2), \cdots m_{t}\left(d_{1}\right)\right] \in \mathbb{R}^{d_{1}}, \\
& \mathbf{n}_{t}=\left[n_{t}(1), n_{t}(2), \cdots n_{t}\left(d_{2}\right)\right] \in \mathbb{R}^{d_{2}},
\end{aligned}
$$

where $d_{2}$-dimensional features $\mathbf{n}_{t}$ are computed from $\mathbf{m}_{t}, d=$ $d_{1}+d_{2}$, and $m_{t}(1) \triangleq G_{t}$ is the CGM data at time $t$. Then the joint probability of the forecasting glucose, $p\left(\mathbf{G}^{\prime}\right)$, is factorised as a product of conditional probabilities as follows

$$
p\left(\mathbf{G}^{\prime}\right)=\prod_{t=1}^{T-w} p\left(G_{t+w}^{\prime} \mid G_{1}, \cdots, G_{t}\right),
$$

where $w$ represents the number of glycemic samples in terms of the $\mathrm{PH}$, and the forecasting glucose sample $G_{t+w}^{\prime}$ is therefore conditioned on the glucose samples at all previous timesteps. Specifically, the CGM measures the glucose every 5 minutes. If we forecast the glucose of next 30 minutes indicating $\mathrm{PH}=30$, then we set $w=30 / 5=6$ samples. Note that 30 mins $\mathrm{PH}$ has been widely used in the literature [8] and applied in the Medtronic MiniMed 640G insulin pump. Because $P i$ (plasma insulin estimation) and $R a$ (glucose rate of appearance) relate to glucose dynamics $\mathbf{G}$ in addition to meal $\mathbf{M}$ and insulin $\mathbf{I}$, for instance, we may set $d_{1}=3, d_{2}=2$ where $\mathbf{m}_{t} \triangleq\left[G_{t}, M_{t}, I_{t}\right]$ and $\mathbf{n}_{t} \triangleq\left[P i_{t}, R a_{t}\right]$, where $\left\{P i_{t}, R a_{t}\right\}$ can be calculated from $\left\{G_{t}, M_{t}, I_{t}\right\}$ and other information of subjects such as body weight [23]. The factors in consideration in $\mathbf{x}$ can be changed accordingly, depending on the data we can collect.

In both training and inference, multi-dimensional health data are sent to the network as batches determined by a sliding window. GluNet uses a stack of dilated convolutional layers to model the conditional probability distribution. We set the outputs of GluNet as a classification over the next forecasting value $G_{t+w}^{\prime}$ targeting at the value $G_{t+w}$ with a softmax layer. It is to maximize the log-likelihood of the data with regard to the parameters

$$
\max \log \left\{\prod_{t=1}^{T-w} p\left(G_{t+w} \mid \mathbf{x}_{1}, \cdots, \mathbf{x}_{t}\right)\right\} .
$$

After the training phase, the forecasting glucose $G_{t+w}^{\prime}$ 's distribution is computed for inference. We tune the hyperparameters on a validation set to avoid overfitting or underfitting carefully.

\section{B. The Architecture of GluNet}

GluNet has four components. They are preprocessing, label transforms and recover, multi-layers of dilated convolutions, postprocessing. In the preprocessing, we clean the data $\mathbf{m}_{t}$, compute useful $\mathbf{n}_{t}$, and prepare them for the DNN use. The label transform component converts the glucose $G$ to the difference $\triangle G$ between current glucose $G_{t}$ and $\mathrm{PH}$ glucose $G_{t+w}$. While in the inference, forecasting glucose is recovered from $\triangle G$. The main ingredients of GluNet are multi-layers of dilated convolutions and gated activations. A stack of dilated convolutional layers are placed. Both residual and parameterised skip connections are applied throughout the network aiming at speeding up the convergence. In addition, we calculate the dilated $\mathrm{CNN}$ in a faster manner by eliminating redundant operations. Fig 2 shows the architecture of GluNet.

\section{Preprocessing}

A preprocessing component is designed to clean the input data. There are several steps, P1: ruling out outliers, P2, P3: interpolation/extrapolation, P4: computing features, P5: alignment. The specific approaches of preprocessing depend on the specific dataset we have. 1) Rule out outliers: The outliers come from the error in CGM measurements, the transmission of CMG data, or incorrect behaviours when people record the data (meal, insulin). These outlier data points 


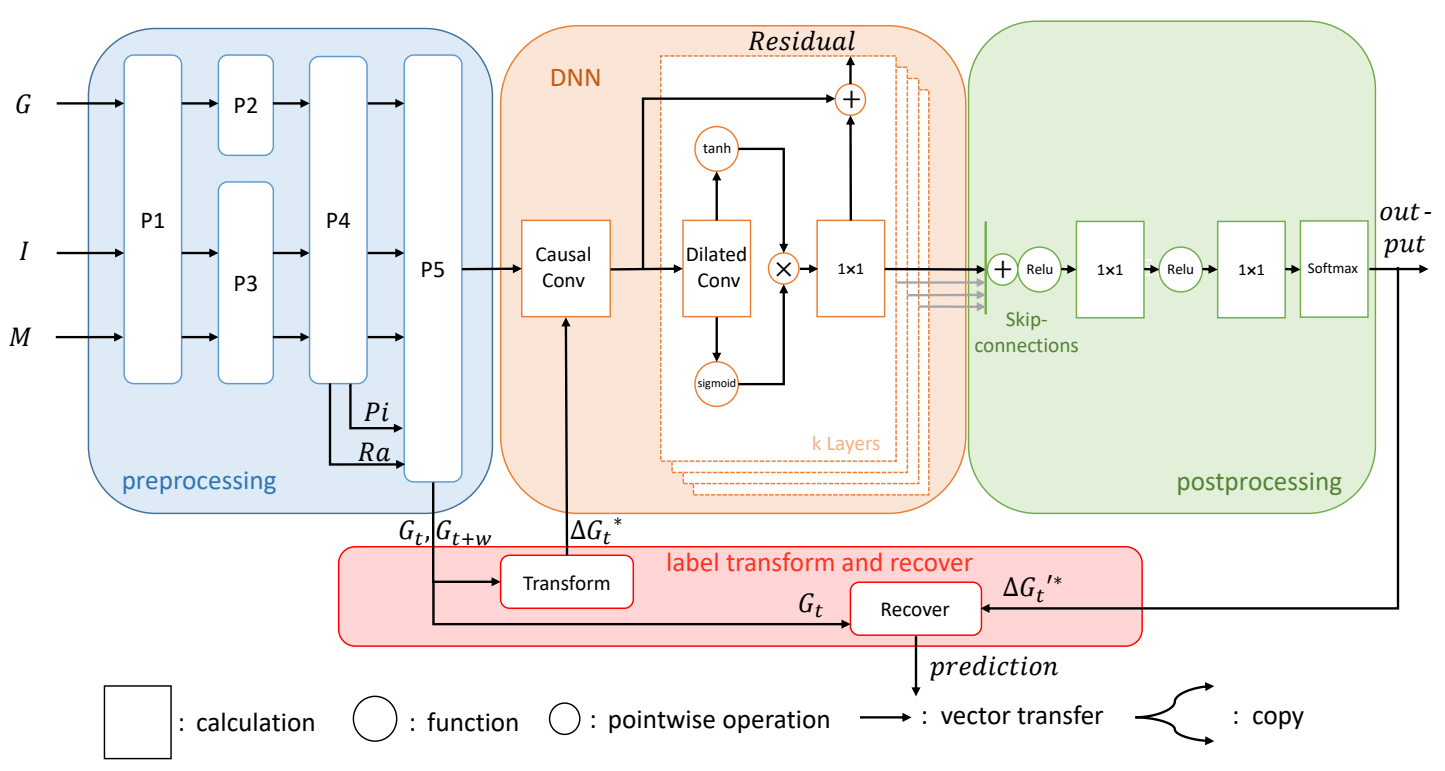

Fig. 2: The architecture of GluNet. It consists of four parts: preprocessing, DNN, postprocessing and label transform and recover. The input data are CGM measurements time series $G$, insulin $I$ and meal $M$. More input factors are optional. In the preprocessing, P1: rule out outliers in $G, I, M$; P2: interpolation of $G$ when the missing data gap is not large; P3: fill or estimate the missing data in $I, M$; P4: calculate other factors as input to the $\mathrm{DNN}$, for instance plasma insulin estimation $P i$ and glucose rate of appearance $R a$; P5: align all factors with the same timeline and use them as input to the DNN. The aligned BG time series $G_{t}$ is also sent to the label transform, and quantized $\triangle G_{t}^{\prime}$ is used as the category target in training.

are supposed to be ruled out. In GluNet we set thresholds in the highest, lowest, and largest differential of the CGM measurements. Sometimes certain meal or insulin values in $M$ or $I$ are too large or too small, which implies that they might be mistakes. We identify these values by threshold method and correct them appropriately. 2) Interpolation/extrapolation: Spline interpolation or extrapolation technique are used when the missing CGM data are less than one hour (12 samples). Spline interpolation is implemented because it is more accurate, and simpler than some other methods [24]. If the missing CMG data are longer than 1 hour, we consider it as a separate dataset in training. In the inference, extrapolation is adopted because future samples cannot be used in forecasting. 3) Considering other factors (optional): Given $\{I, M\}$, we utilize the insulin absorption model and the glucose absorption model proposed in [23] to compute $R a$ and $P i$ based on the estimation technique proposed by Hovorka in [25], where $R a$ denotes the glucose rate of appearance, $P i$ represents the plasma insulin estimation. More factors can be incorporated optionally. 4) Alignment: All data are aligned to $G$ to generate multi-channel data that have the same length of $G$.

\section{Label Transform and Recover}

Aiming at increasing the predictive accuracy, a label transform component is placed before DNN. Rather than directly forecasting $G_{t+w}$ we forecast the difference between $G_{t+w}$ and $G_{t}$ denoted as $\triangle G_{t}=G_{t+w}-G_{t}$ in the training. An index of quantized $\triangle G_{t}$, denoted as $\triangle G_{t}^{*}$, is used as the output label. Without loss of generality the dimension of the output labels is set as 256 . It represents that $\triangle G_{t}$ varies from -12.7 to $12.8 \mathrm{mg} / \mathrm{dl}$, where each label denotes a quantized index $\triangle G_{t}^{*}$ with a quantizing interval of $0.1 \mathrm{mg} / \mathrm{dl}$. Specifically, the label index $\triangle G_{t}^{*}$ can be calculated as

$$
\triangle G_{t}^{*}=\left\{\begin{array}{rlll}
1 & \text { for } & \triangle G_{t}<-12.75 \\
\mathcal{R}\left(10\left(\triangle G_{t}+12.8\right)\right) & \text { for } & -12.75<\triangle G_{t}<12.85 \\
256 & \text { for } & \triangle G_{t}>12.85
\end{array}\right.
$$

where $\mathcal{R}$ denotes a round quantization operator that converts a decimal to an integer. In this way the predictive task is converted to a classification task: given previous data, the model computes the forecasting probability distribution of $\triangle G_{t}$. GluNet forecasts the future glucose as the inference of the DNN by recovering $\mathrm{BG}$ values from label indexes. It is a reverse process of the previous label transform. Firstly $\triangle G_{t}^{\prime}$ is computed using a reverse equation of (4) from inference value $\triangle G_{t}^{\prime *}$. Then the forecast $G_{t+w}^{\prime}$ can be derived from $G_{t+w}^{\prime}=\triangle G_{t}^{\prime}+G_{t}$ where $G_{t}$ is the current glucose.

\section{E. Dilated CNN}

$\mathrm{CNN}$ is a feed-forward artificial neural network, and the calculation that performs in each layer is convolution. In GluNet we use the multi-layer convolutional NN to build the model. A visualization of a stack of causal convolutional layers is shown in Fig. 3 (I), where the forecast at timestep $t$ depends on previous inputs. In PixelCNN, the convolution operator is equivalent to implementing a mask on image pixels by calculating an elementwise multiplication of the mask with the convolution kernel. For time series one can implement it by shifting output of normal convolutions. Because the model has no recurrent connections, the training process is faster than neural networks with recurrent structure. It is important for training long time series. One problem of the casual CNN is 

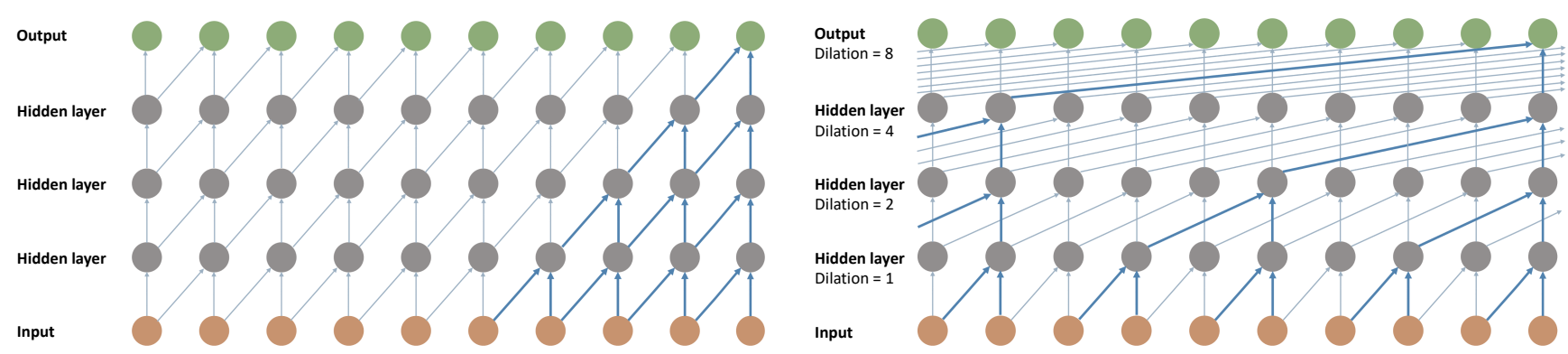

Fig. 3: Visualization of a stack of causal convolutional layers and dilated convolutional layers, where each has 3 hidden layers, orange and green notes represent input and output, respectively. (I) A stack of causal convolutional layers with a receptive field of 5. (II) A stack of dilated convolutional layers with a receptive field of 16 .

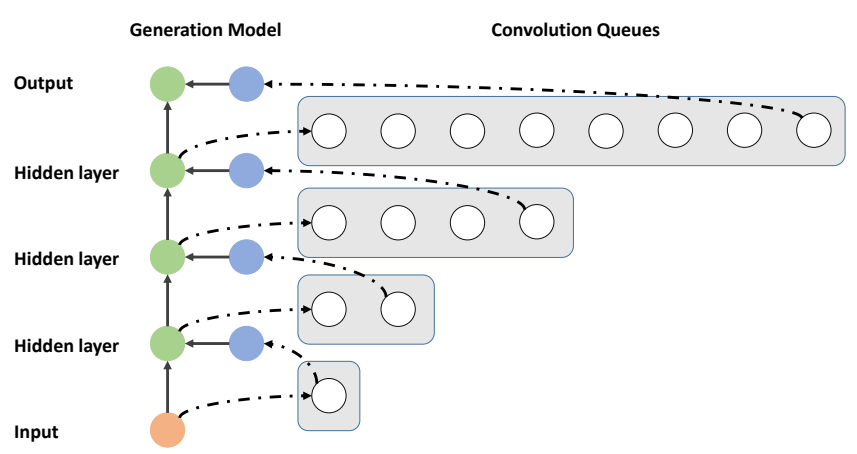

Fig. 4: The diagram of fast dilations. The generation model is initialized with weights from the previous network. After initialization we repeat the pop and push phase iteratively.

that it has a narrow receptive field. Its number of receptive field is similar to the number of layers. To solve it we utilize dilated convolutions to increase the receptive field without significantly increasing computational cost. The key idea of dilated CNN is that it skips the input values in each layer with certain steps, which allows the network to operate on a larger scale that increases the receptive field by orders of the number of layers. As shown in Fig. 3 (II), the dilation is $\{1,2,4,8\}$, while in casual $\mathrm{CNN}$ the dilation remains $\{1,1,1,1\}$.

Moreover, to accelerate the computation, we implement dilated CNN in an efficient manner. Popping and pushing recurrent states in the cache are applied to eliminate redundant convolution operations [26]. It reduces computations from $O\left(2^{L}\right)$ to $O(L)$, where $L$ is the number of layers. Fig. 4 illustrates how it works when dilation is doubled in each layer. Specifically, in the pop phase the convolution queue pops the recurrent state and feeds it to the corresponding state. Then the new hidden states and the output are calculated. While in the push phase, the algorithm pushes the new hidden state into the convolution queue of the above layer. After that, a softmax operator is leveraged to impose the non-linearity using a $\mu$ law transformation [19] to produces a better reconstruction $f\left(x_{t}\right)-\operatorname{sign}\left(x_{t}\right) \frac{\left.1+\mu\left|x_{t}\right|\right)}{\ln (1+\mu)}$, where $\left|x_{t}\right|<1$ and $\mu=255$.

\section{F. Postprocessing}

The postprocessing consists of skip connections and a series of operations. Please refer to Appendix for details. Postprocessing and dilated CNN form the DNN part of GluNet.

\section{RESULTS}

\section{A. Dataset and Model}

1) In silico Data: The adult and adolescent virtual subjects from the UVA/Padova T1D simulator were employed. We generated 180 days data with 3 meals per day for the 10 virtual adult and 10 virtual adolescent subjects. The generated glucose dataset is a time series with 288 points per day. The intra-day variability is applied in meal size [27] with variability $10 \%$, meal time with $S T D=60$ mins and meal amount misestimation [-0.3,0.2]. Furthermore, 30 minutes of exercise with time variability $10 \%$ is considered for 2 days per week, and its time varies in the morning, afternoon or evening. Insulin data has several entries per day. The time of insulin entries can be the same as a meal time, or at different time. These settings are variant enough for simulating subjects' daily data under different conditions. Each dataset was randomly divided into two parts: training set and testing set, where each accounted for $50 \%$ of the dataset. In the training set, $90 \%, 10 \%$ partition is set as the training/validation strategy.

2) Clinical Data: There are two clinical datasets. The first one was obtained from our ABC4D project [21]. T1D diabetic subjects participated and were monitored for 6 consecutive months with Dexcom CGM devices (San Diego, CA). Information on meals, insulin dosages were recorded by the patients themselves through a dedicated App. Similar to the simulated data, each clinical dataset was randomly divided into training set and testing set, where each accounted for around 90 days (50\%) of the dataset. The second dataset was provided by the Blood Glucose Level Prediction Challenge, namely the OhioT1DM dataset [22]. During eight weeks' period, six T1D subjects wore CGM devices and insulin pumps and reported their data regularly. A smart-phone app and a fitness band were used to collect daily events. OhioT1DM dataset is different from other datasets, because its training set and the testing set have been separated by the Challenge, for around 40 days for training and 10 days for testing, respectively.

3) Model Structure and Comparison: In the model framework we use a CNN with 5 layers. For the first three layers, the hidden units are set to 32 , while the top two layer has 64 neurons. We use the sliding window of size 16 time-steps to derive one forecast sample for the future glucose with a many-to-one structure. The inputs are glucose, meal, insulin and time stamps. Only these information are used because 
TABLE I: Prediction performance for the considered prediction methods evaluated on the 10-adult virtual subjects.

\begin{tabular}{|c|c|c|c|c|c|c|c|c|c|}
\hline $\mathrm{PH}$ & Methods & RMSE $(m g / d L)$ & MARD (\%) & Time lag (mins) & $\mathrm{PH}$ & Methods & $\operatorname{RMSE}(m g / d L)$ & MARD (\%) & Time lag (mins) \\
\hline \multirow{5}{*}{$\begin{array}{l}\stackrel{\Xi}{\Xi} \\
\text { 恋 } \\
\text { o }\end{array}$} & GluNet & $\mathbf{8 . 8 8} \pm \mathbf{0 . 7 7}$ & $5.32 \pm 0.66$ & $\overline{\mathbf{0 . 8 3}} \pm \mathbf{0 . 4 0}$ & \multirow{5}{*}{ 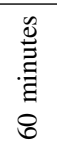 } & GluNet & $19.90 \pm 3.17$ & $\mathbf{1 0 . 5 5} \pm 1.40$ & $\mathbf{1 6 . 4 3} \pm 4.07$ \\
\hline & NNPG & $13.55 \pm 1.30 \ddagger$ & $7.55 \pm 1.10^{\ddagger}$ & $10.12 \pm 1.67^{\dagger}$ & & NNPG & $24.69 \pm 3.19^{\ddagger}$ & $13.39 \pm 2.10^{\ddagger}$ & $30.92 \pm 5.11^{\ddagger}$ \\
\hline & SVR & $14.65 \pm 1.78^{\mp}$ & $7.58 \pm 1.05^{\mp}$ & $11.55 \pm 2.00^{\mp}$ & & SVR & $25.34 \pm 3.71^{\mp}$ & $13.16 \pm 1.89^{\mp}$ & $32.62 \pm 4.35^{\mp}$ \\
\hline & LVX & $12.25 \pm 1.40^{\ddagger}$ & $7.29 \pm 1.02^{\ddagger}$ & $6.54 \pm 2.08^{\ddagger}$ & & LVX & $22.41 \pm 2.89^{\ddagger}$ & $14.06 \pm 2.76^{\ddagger}$ & $35.79 \pm 2.79^{\ddagger}$ \\
\hline & ARX & $13.26 \pm 1.25^{\mp}$ & $7.88 \pm 1.24^{\mp}$ & $9.63 \pm 1.64^{7}$ & & ARX & $25.73 \pm 3.41^{\mp}$ & $14.71 \pm 2.68^{\mp}$ & $40.18 \pm 2.71^{\mp}$ \\
\hline
\end{tabular}

TABLE II: Prediction performance for the considered prediction methods evaluated on the 10-adolescent virtual subjects.

\begin{tabular}{|c|c|c|c|c|c|c|c|c|c|}
\hline $\mathrm{PH}$ & Methods & RMSE $(m g / d L)$ & MARD (\%) & Time lag (mins) & $\mathrm{PH}$ & Methods & RMSE $(m g / d L)$ & MARD (\%) & Time lag (mins) \\
\hline \multirow{5}{*}{ 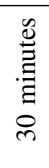 } & GluNet & $10.73 \pm 1.66$ & $5.77 \pm \mathbf{0 . 8 8}$ & $1.44 \pm 0.72$ & \multirow{5}{*}{ 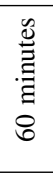 } & GluNet & $22.65 \pm 4.71$ & $11.23 \pm 1.96$ & $12.81 \pm 2.86$ \\
\hline & NNPG & $16.72 \pm 3.18 \ddagger$ & $9.25 \pm 2.60^{\mp}$ & $9.86 \pm 1.99^{\dagger}$ & & NNPG & $32.58 \pm 7.43^{\ddagger}$ & $16.55 \pm 2.83^{\ddagger}$ & $31.69 \pm 4.17^{\ddagger}$ \\
\hline & SVR & $19.72 \pm 4.86^{\ddagger}$ & $8.88 \pm 1.48^{\ddagger}$ & $12.20 \pm 2.15^{\ddagger}$ & & SVR & $34.62 \pm 8.54^{\ddagger}$ & $16.29 \pm 2.49^{\ddagger}$ & $33.80 \pm 4.45^{\ddagger}$ \\
\hline & LVX & $14.99 \pm 3.20^{\mp}$ & $8.07 \pm 1.24^{\mp}$ & $7.36 \pm 1.94^{\ddagger}$ & & LVX & $31.08 \pm 7.78^{\ddagger}$ & $15.31 \pm 2.41^{\ddagger}$ & $26.48 \pm 3.66^{\ddagger}$ \\
\hline & ARX & $15.24 \pm 2.44^{\ddagger}$ & $8.21 \pm 1.37^{\ddagger}$ & $8.16 \pm 1.31^{\ddagger}$ & & ARX & $33.21 \pm 6.84^{\ddagger}$ & $18.57 \pm 3.00^{\ddagger}$ & $38.79 \pm 2.97^{\ddagger}$ \\
\hline
\end{tabular}

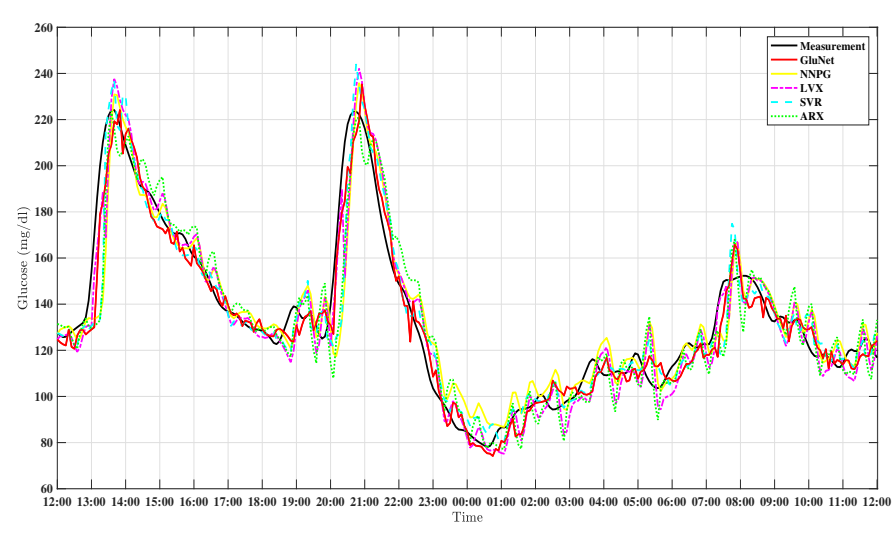

Fig. 5: Virtual adult 2.

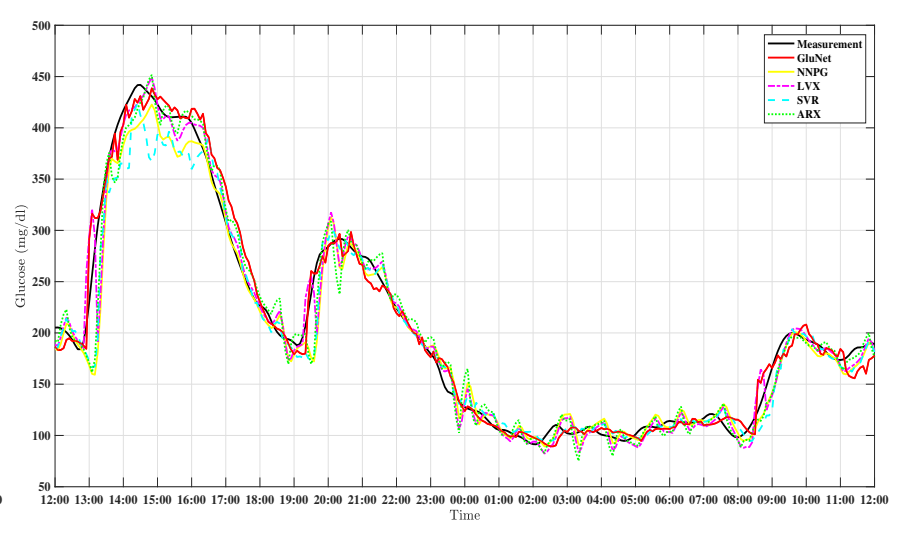

Fig. 6: Virtual adolescent 2

Fig. 7: One day period forecasting results. The solid black line, solid red line, dash-dotted magenta line, dashed cyan line, and dotted green line indicate the simulated glucose measurements, the forecasting results of the GluNet, NNPG, LVX, SVR, and the ARX method, respectively.

we want to have a fair comparison that all methods share the same input data. We compare our results with the neural network for predicting glucose algorithm (NNPG) in [28], the latent variable with exogenous input (LVX) statistical method proposed in [29], the auto regression with exogenous input (ARX) method [30], and the support vector regression (SVR) [12] method. 30 and 60 minutes PH are considered.

\section{B. Evaluation metrics}

The efficiency and accuracy of our proposed method will be demonstrated with the root mean square error (RMSE) results in $m g / d L$ and the mean absolute relative difference (MARD) in $\%$, calculated by

$\mathrm{RMSE}=\sqrt{\frac{1}{N} \sum_{k=1}^{N}\left(y_{k}-\hat{y}_{k}\right)^{2}}, \quad$ MARD $=\frac{1}{N} \sum_{k=1}^{N} \frac{\left|y_{k}-\hat{y}_{k}\right|}{y_{k}}$,

where $y$ denotes the CGM measurement and $\hat{y}$ denotes the forecasting results, $N$ is the total data numbers. Also a prediction time lag (or called prediction time delay [28]) is computed to measure the time-shift between the actual and predicted signal which gives the highest cross correlation coefficient between them:

$$
\tau_{\text {delay }}=\underset{k}{\arg \max }\left(\hat{y}_{k}(k \mid k-P H) \star y(k)\right) .
$$

The prediction time lag provides an accurate estimate of the delay (or offset) in predictions [31]. We use these three criteria because they are widely used in evaluating the glucose forecast in the literature.

\section{In Silico Results}

Table I demonstrates the forecasting performance of the proposed GluNet, NNPG, LVX, SVR, and ARX algorithm, in terms of the corresponding mean values and standard deviations (STD) of RMSE ( $\mathrm{mg} / \mathrm{dL}$ ), MARD (\%) for the 10 adults from the UVA/Padova T1D simulator. Table II demonstrates the forecasting performance for the 10 adolescents from the UVA/Padova T1D simulator, with a same setting to Table II.

From Table II. we can see that GluNet has the smallest RMSE and MARD. The mean value of RMSE are much improved (GluNet $8.88 \pm 0.77$ v.s. others best $12.25 \pm 1.40$ for $\mathrm{PH}=30 \mathrm{mins}$ ). This verifies GluNet's superiority that it performs well for virtual adult subjects. Also the shortest Time lag shows that the algorithm can discover the glucose change 
TABLE III: Forecasting performance for the methods evaluated on the 10 ABC4D adult subjects.

\begin{tabular}{|c|c|c|c|c|c|c|c|c|c|}
\hline $\mathrm{PH}$ & Methods & RMSE $(m g / d L)$ & MARD (\%) & Time lag (mins) & $\mathrm{PH}$ & Methods & RMSE $(m g / d L)$ & MARD (\%) & Time lag (mins) \\
\hline \multirow{5}{*}{ 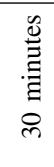 } & GluNet & $19.19 \pm 2.74$ & $\mathbf{1 0 . 4 1} \pm 1.53$ & $11.34 \pm 3.09$ & \multirow{5}{*}{ 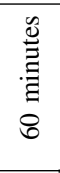 } & GluNet & $31.78 \pm 4.94$ & $19.28 \pm 3.85$ & $30.28 \pm 6.56$ \\
\hline & NNPG & $20.42 \pm 2.76^{*}$ & $11.91 \pm 2.32^{*}$ & $13.88 \pm 3.11 \dagger$ & & NNPG & $33.13 \pm 5.28^{\dagger}$ & $20.59 \pm 4.97^{*}$ & $35.08 \pm 4.49^{*}$ \\
\hline & SVR & $24.96 \pm 4.00^{\mp}$ & $13.04 \pm 2.03 \dagger$ & $14.58 \pm 2.93 \ddagger$ & & SVR & $36.25 \pm 5.95 \ddagger$ & $21.12 \pm 3.77 \ddagger$ & $32.36 \pm 5.09^{\mp}$ \\
\hline & LVX & $21.52 \pm 3.09^{\ddagger}$ & $12.45 \pm 1.95^{*}$ & $15.50 \pm 3.31^{\ddagger}$ & & LVX & $37.34 \pm 6.40^{\ddagger}$ & $20.06 \pm 3.40$ & $41.44 \pm 5.27^{\ddagger}$ \\
\hline & ARX & $21.24 \pm 3.01^{\mp}$ & $12.15 \pm 2.22 *$ & $17.80 \pm 2.35^{\mp}$ & & ARX & $36.17 \pm 5.65^{\mp}$ & $19.85 \pm 3.25$ & $46.29 \pm 2.57^{\mp}$ \\
\hline
\end{tabular}

TABLE IV: Forecasting performance for the considered prediction methods evaluated on the 6 OhioT1DM subjects.

\begin{tabular}{|c|c|c|c|c|c|c|c|c|c|}
\hline & Methods & RMSE $(m g / d L)$ & MARD (\%) & Time lag (mins) & $\mathrm{PH}$ & Methods & RMSE $(m g / d L)$ & MARD (\%) & Time lag (mins) \\
\hline \multirow{5}{*}{ 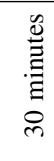 } & GluNet & $19.28 \pm 2.76$ & $\mathbf{8 . 7 3} \pm \mathbf{1 . 7 8}$ & $\mathbf{8 . 0 3} \pm 4.07$ & \multirow{5}{*}{ 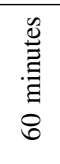 } & GluNet & $\mathbf{3 1 . 8 3} \pm 3.49$ & $\mathbf{1 6 . 1 1} \pm 4.49$ & $\mathbf{1 7 . 7 8} \pm \mathbf{8 . 0 0}$ \\
\hline & NNPG & $22.93 \pm 2.95^{\mp}$ & $10.76 \pm 2.84^{\mp}$ & $16.42 \pm 4.21 \ddagger$ & & NNPG & $35.77 \pm 3.50^{\dagger}$ & $17.75 \pm 4.78^{*}$ & $30.72 \pm 15.28 \ddagger$ \\
\hline & SVR & $21.75 \pm 1.86^{*}$ & $9.23 \pm 2.15$ & $9.86 \pm 4.46$ & & SVR & $34.31 \pm 2.85^{*}$ & $17.86 \pm 4.63^{*}$ & $20.89 \pm 11.40^{*}$ \\
\hline & LVX & $21.70 \pm 3.28^{\ddagger}$ & $9.50 \pm 2.13^{*}$ & $11.75 \pm 5.64^{*}$ & & LVX & $38.95 \pm 12.76^{\ddagger}$ & $17.69 \pm 3.17$ & $27.06 \pm 12.23^{\ddagger}$ \\
\hline & ARX & $21.54 \pm 2.42^{\mp}$ & $9.17 \pm 2.06$ & $11.06 \pm 6.21^{*}$ & & ARX & $39.53 \pm 14.47^{\ddagger}$ & $17.58 \pm 2.98$ & $35.53 \pm 9.38^{\ddagger}$ \\
\hline
\end{tabular}

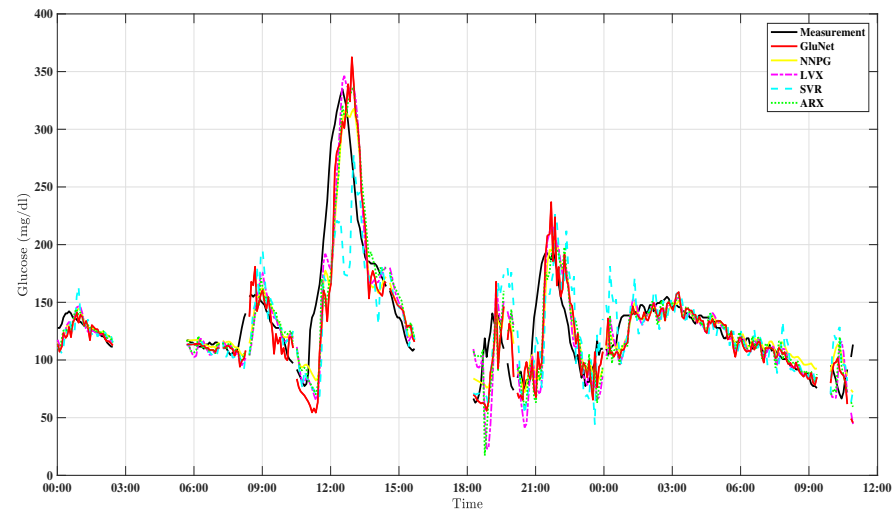

Fig. 8: 1.5 day period forecasting results for adult 2 .

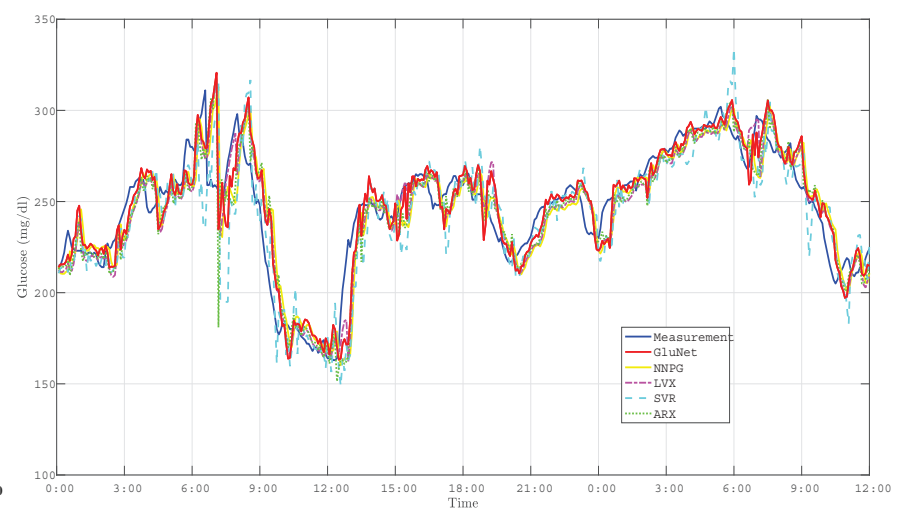

Fig. 9: 1.5 day period prediction results for subject 570 .

Fig. 10: 1.5 day period forecasting results for clinical dataset. The solid black line, solid red line, dash-dotted magenta line, dashed cyan line, and dotted green line indicate the simulated glucose measurements, the forecasting results of the GluNet, NNPG, LVX, SVR, and the ARX method, respectively.

promptly. Meanwhile, the GluNet method achieves the best performance in the case of $\mathrm{PH}=60 \mathrm{mins}$, in terms of RMSE, MARD and Time lag. Fig. 5 demonstrates that GluNet has less oscillation, especially in the rising period after meal intake. In the rising and the dropping stage, forecasting results of GluNet can follow the CGM samples more closely than those of other methods. It explains why GluNet has a small time lag. If we observe the curves, most proportional curves of real and GluNet curves match each other. The overall time lag is computed from the mean of time when it has the highest crosscorrelation over a period of time (e.g. 3 days). If a larger timeshift is made, despite of reducing the time lag near starting points of the rising or dropping stage occasionally, the crosscorrelation of the real and GluNet curves will decrease. That is why the computed time lag is within 1 mins for 30 mins PH. Small time lag improves the hypo-/hyper-glycaemia forecasting performance. Moreover, results for the virtual adolescent group are consist with that for the virtual adults. Smallest mean RMSE, MARD, and Time lag values are achieved, despite of the fact that the adolescent glucose level normally is more difficult to model, as shown in Fig. 6

\section{Clinical Results}

Though In silico results of GluNet are good, it is important that all methods are tested using clinical datasets in practice. As mentioned, two clinical datasets are examined in this section carefully.

1) $A B C 4 D$ Datasets: For the 10 adult subjects from the $\mathrm{ABC} 4 \mathrm{D}$ project and considering 30 and 60 minutes prediction horizon, Table III demonstrates the forecasting performance in RMSE, MARD for three-month test data, corresponding to GluNet, NNPG, LVX, SVR, and the ARX algorithm. Fig. 8 shows 1.5 day period glucose measurements (in solid black line) and the forecasting results of different methods for the subject 2 . The obtained results for clinic data are consistent with that for the in silico data, smaller RMSE and MARD values are achieved for all the subjects and all period time (note that the gap between measurements and curves of all methods near meal time are because of the PH offset). GluNet provides steady forecasting and prompt detection of the glucose change. However, generally most values are much worse than the performances for in silico data. It is because for clinical data, the data has lots of long missing periods, and the real glucose measurement is more complicated affected by factors besides meal, insulin and time. 
2) OhioT1DM Datasets: Table IV presents the 30-minute and 60-minute forecasting performance for six subjects from OhioT1DM datasets in RMSE, MARD, time lag, corresponding to our proposed GluNet, the NNPG, LVX, ARX and the SVR algorithm. The result is leading in the challenge. Fig. 9 shows 1.5 day period glucose measurements (in solid black line) and the prediction results for the subject 570 corresponding to our proposed GluNet method (in solid red line). As comparison, the results of the NNPG (in solid yellow line), LVX (in dash-dotted magenta line), SVR (in dashed cyan line), and ARX method (in dotted green line) are showed.

It is noted that GluNet still has the best RMSE and MARD performance for the OhioT1DM subjects in Table IV] GluNet largely reduces the overall forecasting error and outperforms other methods. Though GluNet performs well, the improvements are not as large as in the last dataset. The reason might be the short length of training set. In ABC4D dataset, 90 days data are used in the training, while in OhioT1DM dataset only 40 days data are available for training. Longer training data usually offers some improvements empirically, in particular when we don't have much data at hand. In Fig. 9, we can see that the prediction curve by GluNet is sensitive to the change of the glucose levels and obtains small time lags, especially at the turning points of the uptrend and downtrend. Therefore, the results are compatible with that for the ABC4D dataset.

Moreover, in type 1 diabetes management the inter- and intra-patient variability are so large that it is difficult to use a generalized model for accurate prediction if no data is available from a new subject. However, in some real clinical scenarios we only have limited historical datasets from several recorded subjects and little information of a new subject. To investigate this problem, we evaluated a generalized GluNet model on new subjects. Particularly, we trained the model on data corresponding to five subjects of ABC4D datasets. Then directly tested it on the rest of subjects one by one. The result turns out that the mean RMSE of generalized model is $24.93 \mathrm{mg} / \mathrm{dl}$ while the personalized model achieves 20.59 $\mathrm{mg} / \mathrm{dl}$, when $\mathrm{PH}=30$ mins. In addition, if limited new data is available, transfer learning can be used to upgrade the generalized GluNet model to a personalized model, like the work [15] and our work [32]. Thus, GluNet can provide useful prediction to new users without much data, and accuracy can be improved as new individual data becomes available. Integrating with cloud, GPUs and mobiles, it provides us with a convenient pattern to train and update models in practice.

\section{Discussion}

\section{A. Advantages and limitations of GluNet}

From the results, we can see that GluNet has several advantages. Firstly, it has a higher forecasting accuracy compared to other existing algorithms (also see the comparison details in the next subsection). Secondly, it is simple to implement, without tedious parameter tuning. Thirdly, it provides richer information of the future glucose level by means of a probabilistic distribution rather than a single value forecasting. Most importantly, GluNet has the shortest time lag and can capture the trend promptly. Here, we implement the proposed

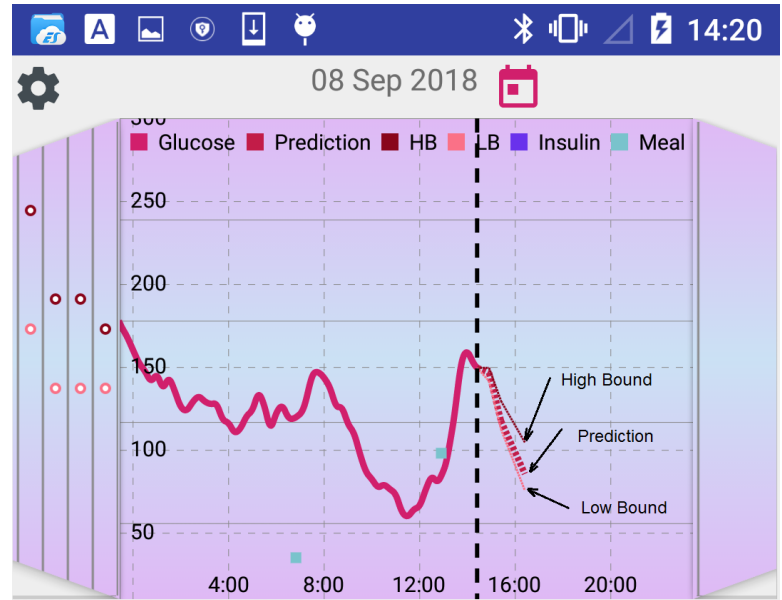

Fig. 11: The display for high and low bounds with the corresponding $\mathrm{PH}$.

algorithm in an Android system on smart phones using TensorLite. The app offers the glucose forecasting as shown in Figure 11. as well as its lower and higher bounds with $95 \%$ probability according to the probabilistic distribution. It uses around $50 \mathrm{MB}$ RAM when sleeping and $100 \mathrm{MB}$ RAM to make the forecasting. The forecasting only takes around $6 \mathrm{~ms}$ in the model inference, which is much faster than running it in Python on a laptop.

One potential limitation of GluNet is that the algorithm heavily relies on the training data quality. In simulation, the data has longer length and has less variability. Thus when we have 6 months high quality data as in the simulation, the forecasting accuracy and time lag can be very good. However, if long data missing period exists, such as in the ABC4D dataset, or the training data is less than two months (in OhioT1DM) with high variability, the performance is significantly affected. In future work, the physiological model could be considered in the DNN model to improve the forecasting performance under these cases.

\section{B. Comparison with other existing algorithms}

We compare GluNet with existing algorithms to show its effectiveness, including NNPG, SVR, LVX, and ARX. Other algorithms published in literature have also been considered. For datasets generated from the simulator, our proposed method is better than [33] $(\mathrm{RMSE}=18.8)$, [34] $(\mathrm{RMSE}=$ 13.7). For real data, GluNet's RMSE 19.2 is better than 23.4 in [35], and 21.8 in [36]. GluNet's time lag 11.3 mins is much better than 20.4 mins in [36] for 30 mins PH. However, due to the unavailability of the original codes and other paper's RMSE, it is not easy to have a fair comparison. We do however try to compare our results with works that have been recently published in the following section.

In [37] the algorithm was compared to ARMA, SVR and doctors on clinical data in terms of RMSE with $\mathrm{PH}=30$ mins. Because we do not have their clinical data, comparing the RMSE directly is not fair. Instead we found that their proposed algorithm is 0.1 better than SVR in terms of RMSE. While GluNet gains 5.7 better than SVR on our clinical data, which implies its advantage. The GluNet's RMSE result of 8.88 for 
virtual adults is also better than the RMSE of 9.40 achieved in [10]. GluNet's result was run on 90 training dates and 90 testing dates, which is more convincing than 11 dates in training and 3 dates in testing from which the result was obtained in [10]. To summarise, GluNet achieves the state-ofthe-art forecasting accuracy with the shortest time lag when compared with published methods.

\section{Parameter Tuning and Training}

For control-theory based algorithms, usually many parameters need to be tuned manually. The forecasting accuracy can be influenced significantly if non-ideal parameters are adopted. In GluNet, several hyper-parameters are set before the training, and these values are fixed for all subjects, such as sliding window size, learning rate followed by the network size. In fact, default learning rate is applied in GluNet. While values of most other parameters are automatically derived via training. At the end of the training, the validation loss is quite close to the training loss, and both loss values are converging. It implies that our model capacity is appropriate and the it shows good generalization of the model. Early stopping technique is used and it prevents the overfitting before running too many epochs. Unlike other glucose forecasting algorithms, GluNet offers a probabilistic distribution of the forecasting glucose over the next $\mathrm{PH}$ period. Thus it provides richer information for the users regarding the future glucose trend.

\section{Computational Cost}

\begin{tabular}{|c|c|c|c|}
\hline Method & Inference (s) & Training (s) & Structure Overview \\
\hline GluNet & 0.79 & $878.11 / 13.26(\mathrm{GPU})$ & 14592 params, 5 layers \\
\hline NNPG & 0.19 & 407.19 & 2196 params, 3 layers \\
\hline SVR & 0.54 & 127.25 & RBF Kernel \\
\hline LVX & 0.05 & 1.73 & 3 PLS LVs, CCA [38 \\
\hline ARX & 0.14 & 4.90 & 3 order \\
\hline
\end{tabular}

TABLE V: Computational time of the methods.

To compare computational cost of GluNet with other methods, we run the models on the subjects from the OhioT1DM datasets using the same hardware. Table V presents the computational time of 10 simulations that train the model on 40-day historical data and then obtain the inference of 10day period. For real-time applications, the inference time is important. Although GluNet consumes more time (878.11s) than other models, all five models have good performance on inference mode (less than one second). Compared with many conventional CNN applications, GluNet has a light structure, yet it still has much more parameters than other considered models. In real-time implementation of such a system, we will train the model on the cloud using GPUs and download the trained model into mobile devices to execute prediction tasks. In this case, it only takes $6 \mathrm{~ms}$ on an Android system to obtain a single prediction for next 30 minutes, and 13.26s on Google Cloud GPU to train the same amount of data.

\section{CONCLUSION AND FUTURE WORK}

In this paper, a glucose forecasting approach called GluNet was proposed using deep neural networks. GluNet achieved better glucose forecasting results in terms of accuracy (RMSE and MARD) and in particular time lag. The proposed approach provides an personalized predictive model by using individual data, and algorithm's computation cost is light enough to be implemented on a mobile phone in real time. GluNet was tested intensively on in silico virtual adult, virtual adolescent and clinical adult datasets with leading performance.

In the future, we consider how to incorporate physiological knowledge in our model. Currently we use them as optional inputs to further improve the its performance.. A better approach is in investigation. We also plan to evaluate GluNet with longer prediction horizons and apply it for over-night hypoglycemia prediction.

\section{APPENDIX}

\section{A. Postprocessing}

If $\mathbf{W}$ denotes the learnable convolution filter,

$$
\mathbf{y}=\tanh \left(\mathbf{W}_{f, k} \star \mathbf{x}\right) \odot \sigma\left(\mathbf{W}_{g, k} \star \mathbf{x}\right),
$$

where $\mathbf{z}$ is the output of unit, $\star, \odot$ are the convolution operator and element-wise multiplication operator, respectively. tanh denotes the tangent function and $\sigma$ denotes the sigmoid function. $f, g, k$ denote filter, gate and layer index. We use the residual in [39] and skip connections throughout the work.

\section{B. Explanation of Time Lag}

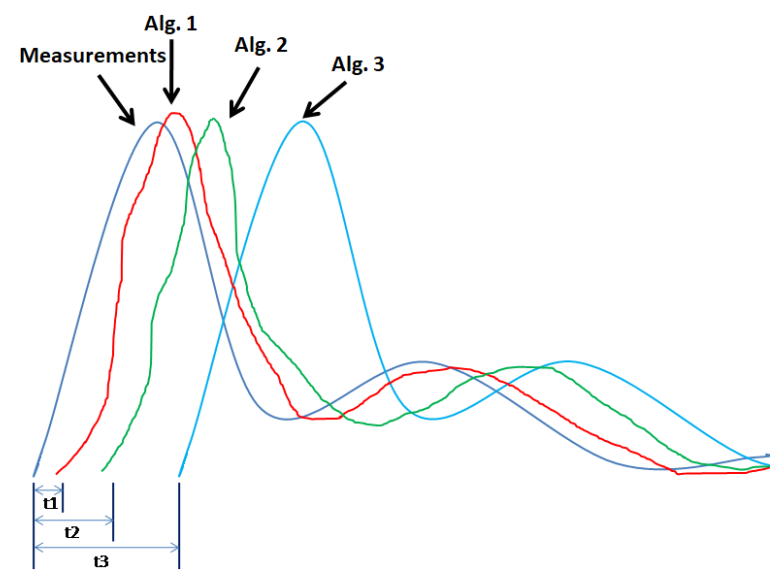

Fig. 12: Graphical representation of the 'prediction time lag'. Consider Alg. 1, Alg. 2 and Alg. 3 (Alg. 3 is to output the current CGM measurements as the prediction result), and the $\mathrm{PH}$ is set to 30 minutes. Then, the time lags of Alg. 1,2,3 are approximately $\mathrm{t} 1, \mathrm{t} 2$ and $\mathrm{t} 3$, respectively, and $\mathrm{t} 3=30$ minutes.

We use Equation (6) to compute the 'prediction time lag' [31], [40]. This definition is different from the 'CGM time lag' that denotes the time lag between the blood glucose and CGM measurements. Fig. 12 shows a diagram of the time lag. In practice it is not easy to obtain the time lag directly, hence the cross correlation is used to compute it. From the example one can see that a shorter time lag generally implies a better prediction, because the algorithm with a shorter time lag captures the curve changes quicker than other algorithms. 


\section{REFERENCES}

[1] K. Ogurtsova, J. da Rocha Fernandes, Y. Huang, U. Linnenkamp, L. Guariguata, N. Cho, D. Cavan, J. Shaw, and L. Makaroff, "Idf diabetes atlas: Global estimates for the prevalence of diabetes for 2015 and 2040," Diabetes Research and Clinical Practice, vol. 128, pp. 40 - 50, 2017.

[2] F. Barcelo-Rico, J. Diez, P. Rossetti, J. Vehi, and J. Bondia, "Adaptive calibration algorithm for plasma glucose estimation in continuous glucose monitoring," IEEE Journal of Biomedical and Health Informatics, vol. 17, no. 3, pp. 530-538, May 2013.

[3] A. Facchinetti, "Continuous glucose monitoring sensors: Past, present and future algorithmic challenges," Sensors, vol. 16, no. 12, 2016.

[4] T. Koutny and M. Ubl, "Parallel software architecture for the next generation of glucose monitoring," Procedia Computer Science, vol. 141, pp. 279-286, 2018.

[5] M. Vettoretti, A. Facchinetti, G. Sparacino, and C. Cobelli, "Type 1 diabetes patient decision simulator for in silico testing safety and effectiveness of insulin treatments," IEEE Transactions on Biomedical Engineering, pp. 1-1, 2018

[6] P. Pesl, P. Herrero, M. Reddy, M. Xenou, N. Oliver, D. Johnston, C. Toumazou, and P. Georgiou, "An advanced bolus calculator for type 1 diabetes: System architecture and usability results," IEEE Journal of Biomedical and Health Informatics, vol. 20, no. 1, pp. 11-17, Jan 2016.

[7] K. Li, F. Liu, H. Dong, P. Herrero, Y. Guo, and P. Georgiou, "A deep learning platform for diabetes big data analysis," in 11th Advanced Technologies and Treatments for Diabetes. Mary Ann Liabery, 2018, pp. A116-A116.

[8] S. Oviedo, J. Vehi, R. Calm, and J. Armengol, "A review of personalized blood glucose prediction strategies for T1DM patients," Int. Journal for Numerical Methods in Biomed. Eng., vol. 33, no. 6, p. 2833, 2017.

[9] M. Eren-Oruklu, A. Cinar, L. Quinn, and D. Smith, "Estimation of future glucose concentrations with subject-specific recursive linear models," Diabetes Tech. and Therapeutics, vol. 11, no. 4, pp. 243-253, Apr. 2009.

[10] C. Zecchin, A. Facchinetti, G. Sparacino, G. D. Nicolao, and C. Cobelli, "Neural network incorporating meal information improves accuracy of short-time prediction of glucose concentration," IEEE Transactions on Biomedical Engineering, vol. 59, no. 6, pp. 1550-1560, Jun. 2012.

[11] C. Zhao, E. Dassau, L. Jovanovic, H. C. Zisser, I. Francis J. Doyle, and D. E. Seborg, "Predicting subcutaneous glucose concentration using a latent-variable-based statistical method for type 1 diabetes mellitus," Journal of Diabetes Science and Technology, vol. 6, no. 3, pp. 617-633, 2012, pMID: 22768893.

[12] E. I. Georga, V. C. Protopappas, D. Ardig, M. Marina, I. Zavaroni, D. Polyzos, and D. I. Fotiadis, "Multivariate prediction of subcutaneous glucose concentration in type 1 diabetes patients based on support vector regression," IEEE Journal of Biomedical and Health Informatics, vol. 17, no. 1, pp. 71-81, Jan 2013.

[13] J. Li and C. Fernando, "Smartphone-based personalized blood glucose prediction," ICT Express, vol. 2, no. 4, pp. 150 - 154, 2016, special Issue on Emerging Technologies for Medical Diagnostics. [Online]. Available: http://www.sciencedirect.com/science/article/pii/S2405959516301126

[14] I. Contreras, S. Oviedo, M. Vettoretti, R. Visentin, and J. Veh, "Personalized blood glucose prediction: A hybrid approach using grammatical evolution and physiological models," PLOS ONE, vol. 12, no. 11, pp. 1-16, 11 2017. [Online]. Available: https: //doi.org/10.1371/journal.pone.0187754

[15] N. M. Rad, S. M. Kia, C. Zarbo, T. van Laarhoven, G. Jurman, P. Venuti, E. Marchiori, and C. Furlanello, "Deep learning for automatic stereotypical motor movement detection using wearable sensors in autism spectrum disorders," Signal Processing, vol. 144, pp. 180-191, 2018.

[16] K. Zarkogianni, K. Mitsis, E. Litsa, M. Arredondo, G. Fico, A. Fioravanti, and K. S. Nikita, "Comparative assessment of glucose prediction models for patients with type 1 diabetes mellitus applying sensors for glucose and physical activity monitoring," Medical \& Biological Engineering \& Computing, vol. 53, no. 12, pp. 1333-1343, Dec. 2015.

[17] H. N. Mhaskar, S. V. Pereverzyev, and M. D. van der Walt, "A deep learning approach to diabetic blood glucose prediction," Frontiers in Applied Mathematics and Statistics, vol. 3, p. 14, 2017. [Online]. Available: https://www.frontiersin.org/article/10.3389/fams.2017.00014

[18] A. van den Oord, N. Kalchbrenner, L. Espeholt, O. Vinyals, and A. Graves, "Conditional image generation with pixelcnn decoders," in Advances in Neural Information Processing Systems. Curran Associates, Inc., 2016, pp. 4790-4798.

[19] A. van den Oord, S. Dieleman, H. Zen, K. Simonyan, O. Vinyals, A. Graves, N. Kalchbrenner, A. Senior, and K. Kavukcuoglu, "Wavenet: A generative model for raw audio," https://arxiv.org/pdf/1609.03499, 2016.

[20] C. D. Man, F. Micheletto, D. Lv, M. Breton, B. Kovatchev, and C. Cobelli, "The uva/padova type 1 diabetes simulator," Jounral of Diabetes Sci Technol., vol. 8, no. 1, pp. 26-34, Jan. 2014.
[21] M. Reddy, P. Pesl, M. Xenou, C. Toumazou, D. Johnston, P. Georgiou, P. Herrero, and N. Oliver, "Clinical safety and feasibility of the advanced bolus calculator for type 1 diabetes based on case-based reasoning: A 6-week nonrandomized single-arm pilot study," Diabetes Technology \& Therapeutics, vol. 18, no. 8, pp. 487-493, 2016, pMID: 27196358. [Online]. Available: https://doi.org/10.1089/dia.2015.0413

[22] C. Marling and R. Bunescu, "The OhioT1DM dataset for blood glucose level prediction," in The 3rd International Workshop on Knowledge Discovery in Healthcare Data, Stockholm, Sweden, July 2018, CEUR proceedings in press, available at http://smarthealth.cs.ohio.edu/bglp/OhioT1DM-dataset-paper.pdf.

[23] P. Herrero, J. Bondia, C. C. Palerm, J. Vehí, P. Georgiou, N. Oliver, and $\mathrm{C}$. Toumazou, "A simple robust method for estimating the glucose rate of appearance from mixed meals," Journal of diabetes science and technology, vol. 6, no. 1, pp. 153-162, 2012.

[24] T. Koutny, "Glucose-level interpolation for determining glucose distribution delay," in XIII Mediterranean Conference on Medical and Biological Engineering and Computing 2013, L. M. Roa Romero, Ed. Cham: Springer International Publishing, 2014, pp. 1229-1232.

[25] R. Hovorka, V. Canonico, L. J. Chassin, U. Haueter, M. Massi-Benedetti, M. O. Federici, T. R. Pieber, H. C. Schaller, L. Schaupp, T. Vering, et al. "Nonlinear model predictive control of glucose concentration in subjects with type 1 diabetes," Physiological meas., vol. 25, no. 4, p. 905, 2004.

[26] T. L. Paine, P. Khorrami, S. Chang, Y. Zhang, P. Ramachandran, M. A. Hasegawa-Johnson, and T. S. Huang, "Fast wavenet generation algorithm," arXiv preprint arXiv:1611.09482, 2016.

[27] C. Dalla Man, R. A. Rizza, and C. Cobelli, "Meal simulation model of the glucose-insulin system," IEEE Transactions on biomedical engineering, vol. 54, no. 10, pp. 1740-1749, 2007.

[28] C. Pérez-Gandía, A. Facchinetti, G. Sparacino, C. Cobelli, E. Gómez, M. Rigla, A. de Leiva, and M. Hernando, "Artificial neural network algorithm for online glucose prediction from continuous glucose monitoring," Diabetes tech. \& therapeutics, vol. 12, no. 1, pp. 81-88, 2010.

[29] C. Zhao, E. Dassau, L. Jovanovič, H. C. Zisser, F. J. Doyle III, and D. E. Seborg, "Predicting subcutaneous glucose concentration using a latentvariable-based statistical method for type 1 diabetes mellitus," Journal of diabetes science and technology, vol. 6, no. 3, pp. 617-633, 2012.

[30] D. A. Finan, F. J. Doyle III, C. C. Palerm, W. C. Bevier, H. C. Zisser, L. Jovanovič, and D. E. Seborg, "Experimental evaluation of a recursive model identification technique for type 1 diabetes," Journal of diabetes science and technology, vol. 3, no. 5, pp. 1192-1202, 2009.

[31] A. Gani, A. V. Gribok, Y. Lu, W. K. Ward, R. A. Vigersky, and J. Reifman, "Universal glucose models for predicting subcutaneous glucose concentration in humans," IEEE Transactions on Information Technology in Biomedicine, vol. 14, no. 1, pp. 157-165, 2009.

[32] J. Chen, K. Li, P. Herrero, T. Zhu, and P. Georgiou, "Dilated recurrent neural network for short-time prediction of glucose concentration," in Proceedings of the 3rd International Workshop on Knowledge Discovery in Healthcare Data (IJCAI-ECAI 2018), Stockholm, Schweden, July 13 , 2018., 2018, pp. 69-73.

[33] G. Sparacino, F. Zanderigo, S. Corazza, A. Maran, A. Facchinetti, and C. Cobelli, "Glucose concentration can be predicted ahead in time from continuous glucose monitoring sensor time-series," IEEE Transactions on Biomedical Engineering, vol. 54, no. 5, pp. 931-937, May 2007.

[34] S. G. Mougiakakou, A. Prountzou, D. Iliopoulou, K. S. Nikita, A. Vazeou, and C. S. Bartsocas, "Neural network based glucose insulin metabolism models for children with type 1 diabetes," in 2006 International Conference of the IEEE Engineering in Medicine and Biology Society, Aug. 2006, pp. 3545-3548.

[35] A. Gani, A. V. Gribok, S. Rajaraman, W. K. Ward, and J. R. * "Predicting subcutaneous glucose concentration in humans: Data-driven glucose modeling," IEEE Transactions on Biomedical Engineering, vol. 56, no. 2, pp. 246-254, Feb. 2009.

[36] Q. Sun, M. V. Jankovic, L. Bally, and S. G. Mougiakakou, "Predicting blood glucose with an lstm and bi-lstm based deep neural network," in 14th Symp. on Neur.l Net. and App. (NEUREL), Nov. 2018, pp. 1-5.

[37] K. Plis, R. Bunescu, C. Marling, J. Shubrook, and F. Schwartz, "A machine learning approach to predicting blood glucose levels for diabetes management," in Modern Artificial Intelligence for Health Analytics Papers from the AAAI-14.

[38] H. Yu and J. F. MacGregor, "Post processing methods (pls-cca): simple alternatives to preprocessing methods (osc-pls)," Chemometrics and intelligent laboratory systems, vol. 73, no. 2, pp. 199-205, 2004

[39] K. He, X. Zhang, S. Ren, and J. Sun, "Deep residual learning for image recognition," IEEE Conference on Computer Vision and Pattern Recognition (CVPR), pp. 770-778, 2016.

[40] E. I. Georga, V. C. Protopappas, and D. I. Fotiadis, "Glucose prediction in type 1 and type 2 diabetic patients using data driven techniques,' Knowledge-oriented applications in data mining, pp. 277-296, 2011. 